\title{
Magnetic breakdown investigated by means of the magnetic-field-containing relativistic tight-binding approximation method
}

\author{
Katsuhiko Higuchi \\ Graduate School of Advanced Sciences of Matter, Hiroshima University, Higashi-Hiroshima 739-8527, Japan \\ Dipendra Bahadur Hamal \\ Department of Natural Sciences (Physics), Kathmandu University, Dhulikhel, Kavre 6250, Nepal \\ Masahiko Higuchi \\ Department of Physics, Faculty of Science, Shinshu University, Matsumoto 390-8621, Japan \\ (Received 26 June 2017; revised manuscript received 6 October 2017; published 15 December 2017)
}

\begin{abstract}
We investigate the magnetic breakdown (MB) phenomena by means of the recently proposed magnetic-fieldcontaining relativistic tight-binding approximation (MFRTB) method [Phys. Rev. B 91, 075122 (2015)]. In the MFRTB method, the MB phenomena can be described as the electron hopping between adjacent semiclassical orbits. It is shown that a set of magnetic energy bands is generated by the MB in the cluster that corresponds to the semiclassical energy level. It is also found that magnetic energy bands originating from the MB and those originating from the semiclassical orbit lying on the constant energy surface are hybridized to each other. Such hybridization leads to various subclusters that correspond to energy states of the so-called forbidden orbits. Magnetic oscillations related to the MB occur when the subcluster changes from the occupied state to unoccupied one and vice versa.
\end{abstract}

DOI: 10.1103/PhysRevB.96.235125

\section{INTRODUCTION}

Magnetic oscillations in metals, such as the de Haas-van Alphen (dHvA) oscillation [1-4] and the Shubnikov-de Haas oscillation [4,5], are widely used to investigate the shape of the Fermi surface, the cyclotron effective mass, and the relaxation time of the conduction electron. Magnetic oscillation phenomena are usually treated on the basis of the semiclassical approximation [4,6-9]. In the usual treatment, which will be referred to hereafter as the semiclassical approach, both the Bohr-Sommerfeld quantization rule that is obtained within the semiclassical approximation and the equation of motion for a Bloch electron in the magnetic field are used to get quantized energy levels (semiclassical energy levels) and electronic orbits [4,6-9]. According to the semiclassical approach, the electron moves in $\boldsymbol{k}$-space along the outline of the cross section of the constant energy surface by a plane perpendicular to the magnetic field [4,6-9]. If electronic orbits in $\boldsymbol{k}$-space are close to each other, the adjacent orbits connect to each other due to tunneling. This is the conventional description of magnetic breakdown (MB) [4,10-15]. Since the orbit changed by the MB (MB orbit) has a different cross section on the Fermi surface, the magnetic oscillation appears with a period that is different from that of the dHvA oscillation [4,10-15]. Hereafter, the magnetic oscillation that is related to the MB orbit will be referred to as the MB oscillation. Analysis of the $\mathrm{MB}$ oscillation is also indispensable for the investigation of the shape of the Fermi surface [4].

There have been other attempts to describe MB oscillations. One is based on the effective-mass approximation [4,8,9]. By means of the effective-mass approximation, it is shown that $\mathrm{MB}$ oscillations originating from so-called forbidden orbitals [4,10-17] are related to the chemical potential oscillation $[16,17]$. Besides this, the Hofstadter method [18] is utilized to describe MB oscillations [19-28]. It is found that the
Hofstadter method can also describe the MB oscillations originating from forbidden orbitals [19-26]. Furthermore, the Hofstadter method is also applied to a honeycomb lattice system, in which the magnetic oscillation is shown to appear even though the area of the Fermi surface vanishes [28].

Recently, we have developed the magnetic-field-containing relativistic tight-binding approximation (MFRTB) method [29]. This method enables us to calculate the energy-band structure of materials immersed in a magnetic field. Hereafter, we refer to the energy bands and energy-band structure of materials immersed in a magnetic field as the magnetic energy bands and magnetic energy-band structure, respectively. It is shown that the MFRTB method provides the modified Hofstadter butterfly diagram if it is applied to the twodimensional square lattice system with $s$-electrons [29]. We have applied this method to crystalline silicon immersed in a magnetic field, and we revealed its magnetic energy-band structure that is defined in the magnetic first Brillouin zone [29]. Also, the MFRTB method can reproduce the dHvA oscillation [30], similarly to the Hofstadter method. It was shown in a previous work [31] that the cluster of the magnetic energy bands, which corresponds to a semiclassical energy level, causes the dHvA oscillation. Furthermore, it is found that the fine energy level structure in the cluster leads to additional oscillation peaks that cannot be explained by the semiclassical approach [31].

As mentioned above, the MFRTB method is reliable for describing the magnetic properties of metals immersed in a magnetic field. Therefore, it is expected that the MFRTB method may describe MB phenomena in a similar way to the Hofstadter method. Although it is shown that MB phenomena can be described by the Hofstadter method, MB phenomena have not yet been discussed on the basis of magnetic energyband structure [19,21-28]. In this paper, after confirming the revisitation of the MB through the MFRTB method, we reveal 


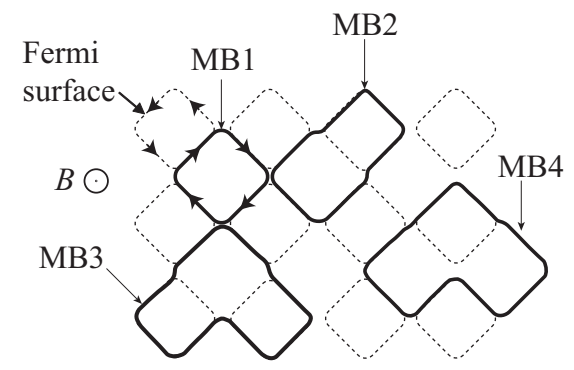

FIG. 1. Schematic view of the Fermi surface (dots line) and electronic orbits containing the MB points (solid lines denoted by MB1, MB2, MB3, and MB4). The arrows denote the direction of the orbital motion for electrons, which is predicted by the semiclassical approach. Note that the MB2, MB3, and MB4 orbits are so-called forbidden orbits, parts of which are incompatible with the direction of the orbital motion predicted by the semiclassical approach.

what kinds of magnetic energy bands are generated by the MB, and we describe how the MB oscillations are produced by such magnetic energy bands.

The outline of this paper is as follows. In Sec. II, it is shown that the MB phenomena can be revisited by the MFRTB method. In Sec. III, we give a description of the MB phenomena through the MFRTB method. In Sec. IV, we identify the magnetic energy bands that are generated by the MB. Then, we discuss the generating mechanism of the MB oscillations on the basis of magnetic energy bands. In Sec. V, we present concluding remarks.

\section{REVISITATION OF MAGNETIC BREAKDOWN THROUGH THE MFRTB METHOD}

The MFRTB method is applied to the two-dimensional square lattice with $s$-electrons immersed in a magnetic field. The $E$ - $\boldsymbol{k}$ curve of the system for the zero-magnetic-field case is given by $E(\boldsymbol{k})=E_{0}-2 t\left\{\cos \left(k_{x} a\right)+\cos \left(k_{y} a\right)\right\}$, where $E_{0}$ and $t$ denote a constant value and a tight-binding parameter, respectively, and $a$ denotes the lattice constant. In this paper, we take -12.1538 and $-1.7391 \mathrm{eV}$ for $E_{0}$ and $t$, respectively [29]. Figure 1 shows the schematic view of the Fermi surface (dots line) and electronic orbits containing the MB points (solid lines denoted by MB1, MB2, MB3, and MB4). According to the semiclassical approach, the oscillation frequency is proportional to the cross-sectional area of the Fermi surface in a plane perpendicular to the magnetic field. In Fig. 1, we show five orbits in order starting with the orbit having the smallest cross-sectional area. If the band filling factor $\eta$ is more (less) than 0.5 , then the Fermi surface is holelike (electronlike). The closer $\eta$ is to 0.5 , the more likely it is that MB occurs because electronic orbits in $\boldsymbol{k}$-space become closer to each other.

The MFRTB method is applied to this system immersed in a magnetic field. The magnitude of the magnetic field is given by $B=\frac{2 \pi \hbar}{e a^{2}} \frac{p}{q}$, where $p$ and $q$ are relatively prime integers [18,29]. For convenience, the outline of the MFRTB method is given in the Appendix. We calculate the magnetic-field dependences of the total energy, and we obtain the magnetization by the numerical derivative of the total energy with respect to the magnetic field. Note that the total number of electrons per unit volume is fixed $[15-17,20]$ in the present calculations.

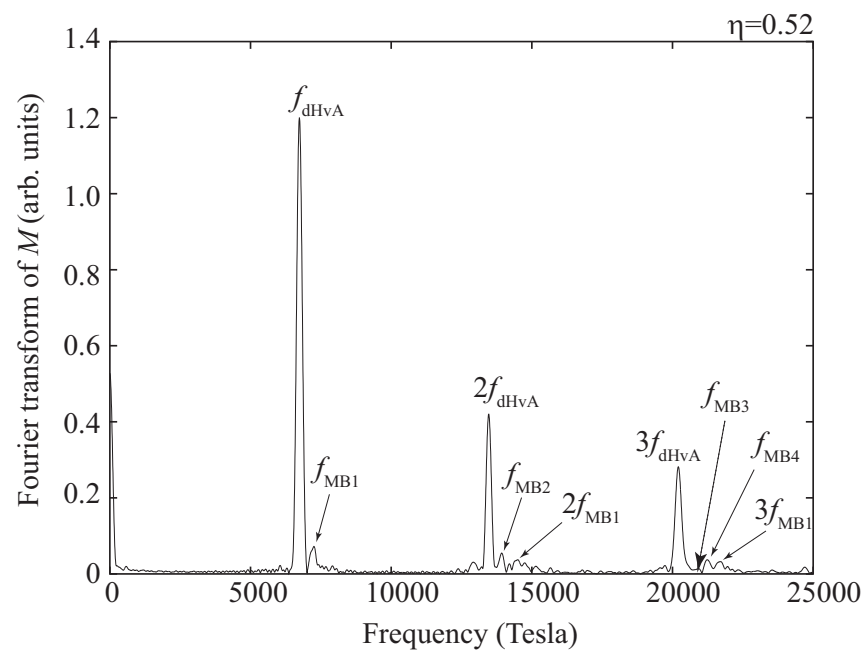

FIG. 2. Fourier transformation of the magnetization for the case of $\eta=0.52$.

Figure 2 shows the coefficient of the Fourier transformation of the magnetization for the case of $\eta=0.52$, where we use the Hamming function as the window function of the Fourier transformation. The positions of the largest peak (denoted by $\left.f_{\mathrm{dHvA}}\right)$ and its higher harmonics $\left(2 f_{\mathrm{dHvA}}, 3 f_{\mathrm{dHvA}}\right)$ just coincide with the frequencies of the dHvA oscillation and its higher harmonics, respectively. It is also confirmed that the positions of other peaks denoted by $f_{\mathrm{MB} 1}, f_{\mathrm{MB} 2}, f_{\mathrm{MB} 3}$, and $f_{\mathrm{MB} 4}$ are identical with frequencies that correspond to orbits of MB1, MB2, MB3, and MB4 (Fig. 1), respectively. Thus, MB oscillations as well as the dHvA oscillation can be reproduced by the MFRTB method.

Next, we shall comment on other peaks that are found in Fig. 2. For instance, we can find a weak peak between $f_{\mathrm{MB} 2}$ and $2 f_{\mathrm{MB} 1}$. Although there are possible orbits other than those shown in Fig. 1, the cross-sectional areas of such orbits are larger than those of the orbits shown in Fig. 1. Therefore, oscillation frequencies of such orbits are out of the range of the horizontal axis of Fig. 2. As a result, weak peaks cannot be explained by the semiclassical approach. One possible explanation regarding the origin of weak peaks is that they come from additional oscillation peaks that are predicted in the previous paper [31]. Another possible explanation is that they are only due to side lobes that are generated in the process of Fourier transformation. More investigations are needed to identify the origin of weak peaks.

\section{DESCRIPTION OF THE MB PHENOMENA THROUGH THE MFRTB METHOD}

In this section, we discuss how the MB phenomena are described by the MFRTB method. In general, the semiclassical electron orbit in real space can be obtained by rotating the semiclassical electron orbit in $\boldsymbol{k}$-space $90^{\circ}$ [8]. Therefore, the electronic orbit involving MB points looks like the rotated orbit of Fig. 1. Since the area of the semiclassical electronic orbit in real space is obtained by multiplying the factor $(\hbar / e B)^{2}$ by that in $\boldsymbol{k}$-space [8], the area of the semiclassical electronic orbit in real space shrinks with increasing $B$. In the MFRTB method, 


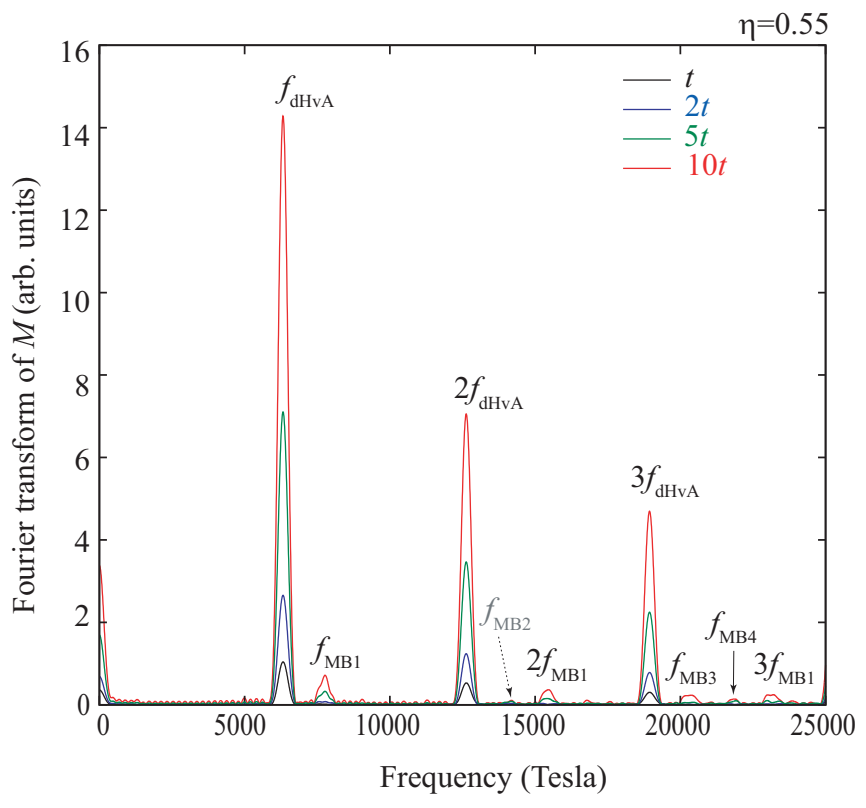

FIG. 3. Dependence of the Fourier transformation of the magnetization on the TB parameter.

the eigenfunction is given by the linear combination of the atomic orbitals immersed in a magnetic field. Judging from the successful revisitation [30,31] of the dHvA oscillation, which is well described by the semiclassical approach, the linear combination represents the orbit that is close to the semiclassical electronic orbit. Therefore, the area of the orbit is expected to shrink with increasing $B$, which may facilitate electron hopping between adjacent orbits [29]. This electron hopping connects adjacent orbits to each other, which leads to the energy width of the energy level [29]. That is, the magnetic energy bands are formed by the electron hopping between adjacent orbits.

On the other hand, the electron hopping between adjacent orbits leads to various kinds of extended orbits. As is expected, extended electronic orbits such as MB1, MB2, MB3, and MB4 would be generated by the electron hopping between adjacent orbits. Figure 3 shows the dependence of the MB oscillation on the TB parameter, which gives evidence for the direct relation between the MB and the electron hopping. It is found from Fig. 3 that the amplitude of the MB oscillation becomes larger as the TB parameter becomes larger. The large TB parameter promotes the electron hopping between adjacent semiclassical orbits. Therefore, the MB is more likely to occur as the TB parameter becomes larger. Thus, the MB phenomena can be recognized as the electron hopping between adjacent semiclassical orbits. In addition, since the electron hopping between adjacent semiclassical orbits leads to the widening of the magnetic energy band, as mentioned above [29], the energy width indicates to what extent the MB likely occurs.

\section{RELATION BETWEEN MB OSCILLATION AND MAGNETIC ENERGY BANDS}

In this section, we mention the relation between $\mathrm{MB}$ oscillations and magnetic energy bands. Figure 4 shows

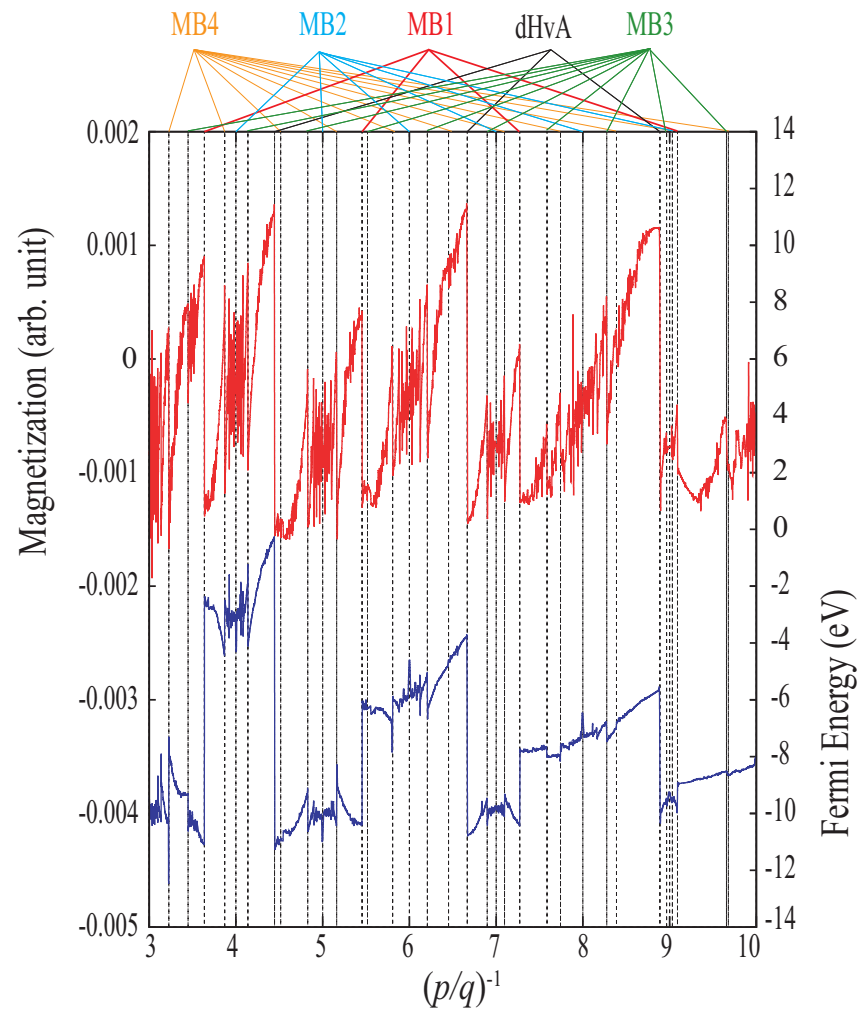

FIG. 4. Dependence of the magnetization and the Fermi energy on the magnitude of the magnetic field $(p / q)$. The vertical dashed lines indicate the positions of the $\mathrm{dHvA}$ and $\mathrm{MB}$ oscillations.

the magnetic-field dependences $[(p / q)$ dependences $]$ of the magnetization and Fermi energy for the $\eta=0.52$ case. Many oscillation peaks are found in Fig. 4. To identify the origin of the oscillation peak, the set of vertical dashed lines that indicates the period of the dHvA, MB1, MB2, MB3, or MB4 oscillations is drawn in Fig. 4. The period is calculated by

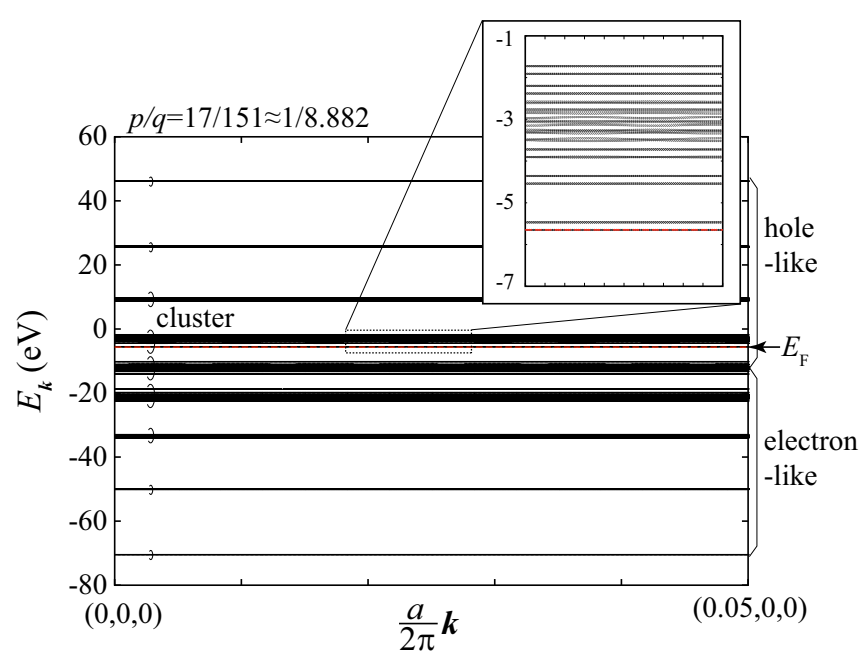

FIG. 5. Overall view of the magnetic energy-band structure for the case of $p / q=17 / 151 \approx 1 / 8.882$. The position of the Fermi energy $\left(E_{F}\right)$ for the case of $\eta=0.52$ is indicated by the red line and arrow. 
$2 \pi e / \hbar A_{\alpha}$, where $A_{\alpha}$ denotes the cross section of the orbit $\alpha$ (=dHvA, MB1, MB2, MB3, or MB4). As shown in Fig. 4, major oscillations come from the $\mathrm{dHvA}, \mathrm{MB} 1, \mathrm{MB} 2, \mathrm{MB} 3$, or MB4. It is also found that the Fermi energy changes rapidly at the vertical dashed lines. This implies that, in a similar way to the dHvA oscillation, magnetic oscillations of MB1, MB2, MB3, and MB4 would occur when some magnetic energy bands become occupied or unoccupied states. With the aid of Fig. 4, the relation between magnetic energy bands and magnetic oscillations of the $\mathrm{dHvA}, \mathrm{MB} 1, \mathrm{MB} 2, \mathrm{MB} 3$, and MB4 is investigated.

Figure 5 shows the overall view of the magnetic energyband structure for the case of $p / q=17 / 151 \approx 1 / 8.882$. The horizontal axis denotes the wave vector defined in the magnetic Brillouin zone [29]. In Fig. 5, we can find nine clusters. Each cluster consists of a lot of magnetic energy bands, as shown in the inset of Fig. 5. The magnetic energy bands are nearly flat, which corresponds to the quantization of the orbital motion of electrons in a uniform magnetic field [29]. As shown in Ref. [31], the dHvA oscillation appears whenever the cluster of the energy bands crosses the Fermi energy with increasing $B$. Therefore, each cluster corresponds to a semiclassical energy level [31]. As is expected from the fact that the semiclassical

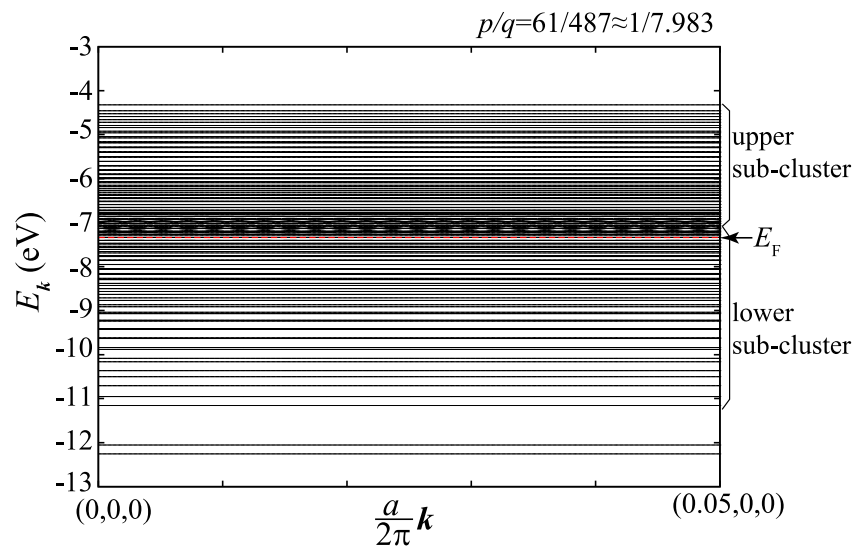

(a)

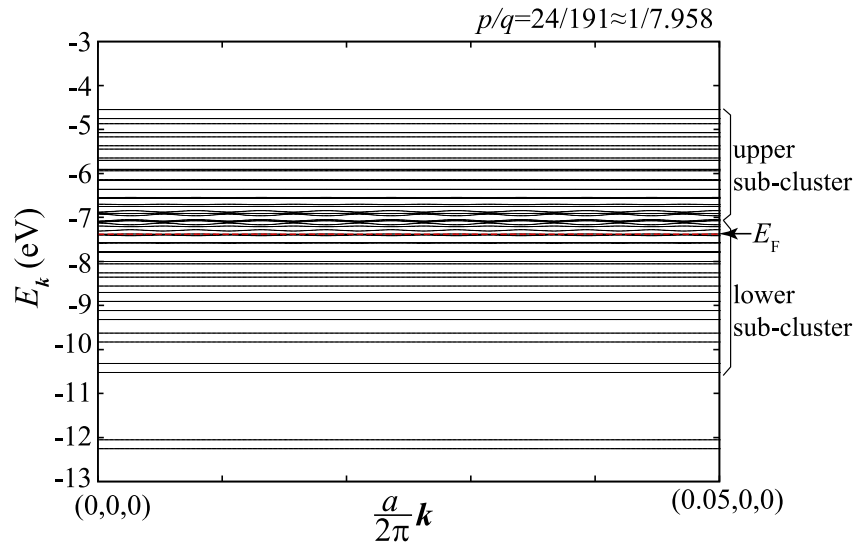

(c) orbit on the constant-energy surface $[E(\boldsymbol{k})=E]$ is a holelike orbit for $E>E_{0}=-12.1538(\mathrm{eV})$, the cluster with a higher energy than the center $\left(E_{0}\right)$ goes down with increasing $B$. On the other hand, the cluster with a lower energy than $E_{0}$ goes up with increasing $B$, corresponding to the fact that the semiclassical orbit with $E<E_{0}$ is an electronlike orbit.

It is found from Fig. 5 that the energy width of the cluster (bandwidth), which corresponds to Harper broadening [32], becomes wider as the position of the cluster approaches the center. This can be understood as follows. The semiclassical orbits in $\boldsymbol{k}$-space are close to each other if the corresponding energy levels lie near the center. Therefore, the electronic hopping between these adjacent semiclassical orbits occurs more readily as the energy levels approach the center. Since the electron hopping between adjacent semiclassical orbitals results in a widening of the bandwidth [29], the bandwidth becomes wider as the position of the cluster approaches the center. Furthermore, it is found from the inset of Fig. 5 that the cluster near the center has a fine energy level structure. That is, the cluster consists of several subclusters. As shown below, these subclusters play an important role in MB oscillations.

With increasing $B$ from the case of Fig. 5, the bandwidth near the center becomes wider. As mentioned below, magnetic

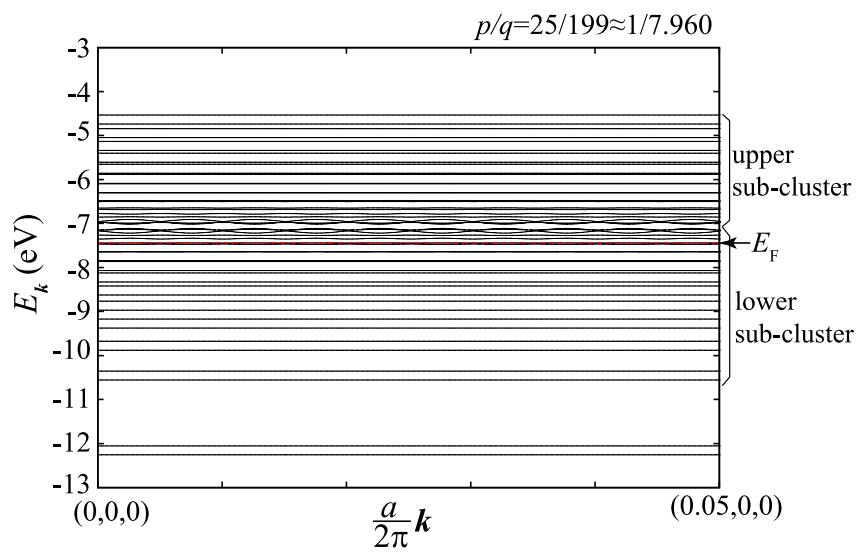

(b)

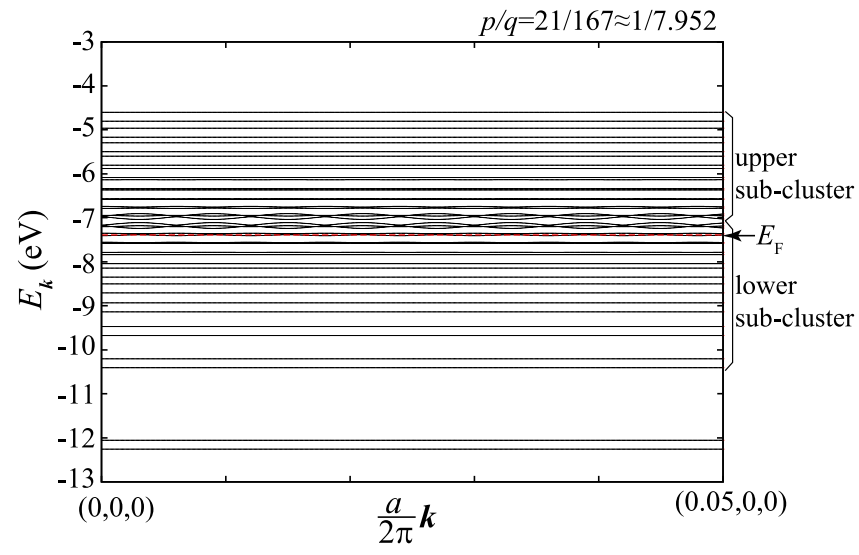

(d)

FIG. 6. Dependence of magnetic energy bands near the center on $p / q$, the values of which are (a) $61 / 487(\approx 1 / 7.983)$, (b) $25 / 199(\approx 1 / 7.960)$, (c) $24 / 191(\approx 1 / 7.958)$, and (d) $21 / 167(\approx 1 / 7.952)$, respectively. The position of the Fermi energy $\left(E_{F}\right)$ for the case of $\eta=0.52$ is indicated by the red line and arrow. 
energy bands that would be caused by the MB appear in the cluster near the center. Figures 6(a)-6(d) show the magnetic-field dependence of magnetic energy bands near the center for higher-magnetic-field cases, the $p / q$ values of which are (a) $61 / 487(\approx 1 / 7.983)$, (b) $25 / 199(\approx 1 / 7.960)$, (c) $24 / 191(\approx 1 / 7.958)$, and (d) $21 / 167(\approx 1 / 7.952)$, respectively. In these figures, the set of magnetic energy bands lying between -11.5 and $-4 \mathrm{eV}$ corresponds to a cluster near the center. The magnetic energy bands in this cluster show a characteristic dependence on the magnetic field. That is, magnetic energy bands are classified into two kinds of subclusters by their magnetic-field dependence. One is that the constituent magnetic energy bands lie in the lower half of the cluster and go up with increasing $B$. The other is that they lie in the upper half of the cluster and go down with increasing $B$. Judging from the magnetic-field dependence, it seems that magnetic energy bands belonging to the former subcluster originate from electronlike semiclassical orbits, while magnetic energy bands belonging to the latter subcluster originate from holelike semiclassical orbits. Considering the fact that electronlike semiclassical orbits on the constant-energy surface exist only below the center, the above-mentioned dependence strongly suggests that the set of magnetic energy bands in the lower half of the cluster originates from the MB1 orbit. This is because (i) the derivative of the cross section $\left(A_{\mathrm{MB} 1}\right)$ with respect to the energy is positive, so that the cyclotron effective mass becomes positive for the MB1 orbit, and (ii) magnetic energy bands of this type appear only near the center where the MB occurs more frequently than other energy ranges. It should be noted that the lower and upper subclusters have a wide energy width. While the widening of the upper subcluster is, as mentioned above, due to the hybridization between semiclassical orbits that is caused by electron hopping between them, that of the lower subcluster is due to hybridization between MB1 orbits that is caused by electron hopping between them.

With increasing $B$, the lower and upper subclusters that are shown in Figs. 6(a)-6(d) approach each other. This would cause the hybridization between the MB1 orbits (lower subcluster) and the semiclassical orbits on the constantenergy surface (upper subcluster). Figures 7(a)-7(h) show the magnetic-field dependence of magnetic energy bands for higher-magnetic-field cases, the $p / q$ values of which are (a) $59 / 457(\approx 1 / 7.746)$, (b) $25 / 193(\approx 1 / 7.720)$, (c) $26 / 199$ $(\approx 1 / 7.654)$, (d) $25 / 191(\approx 1 / 7.640)$, (e) $50 / 379(\approx 1 / 7.580)$, (f) $24 / 179(\approx 1 / 7.458),($ g) $29 / 211(\approx 1 / 7.276)$, and $(\mathrm{h})$ $48 / 349(\approx 1 / 7.270)$, respectively. As shown in Fig. 7(a), three subclusters are formed around $-7 \mathrm{eV}$ as a consequence of the hybridization of the lower and upper subclusters of Figs. 6(a)-6(d). The middle subcluster out of three passes through the Fermi energy with increasing $B$ [Fig. 7(b)], and thus becomes an unoccupied state. Corresponding to this change, the Fermi energy goes down from the middle subcluster to the lowest one [Fig. 7(b)]. At this magnetic field $[p / q=25 / 193(\approx 1 / 7.720)]$, the MB oscillation related to the MB4 orbit appears as shown in Fig. 4. This implies that magnetic energy bands of the middle subcluster are related to the MB4 orbit.

The highest subcluster in Fig. 7(b) and the middle subcluster (MB4 orbit) overlap each other with increasing $B$, so that two subclusters appear in the case of Fig. 7(c). Magnetic energy bands in the lower subcluster, which is denoted by MB3 orbit in Fig. 7(c), are partially occupied in the case of Fig. 7(c). As shown in Figs. 7(d) and 7(e), the number of magnetic energy bands in the lower subcluster decreases with increasing $B$, so that magnetic energy bands in the lower subcluster are fully occupied. In response to this change, the Fermi energy goes up from the lower subcluster to the upper subcluster [Fig. 7(e)]. Since the oscillation peak related to the MB3 orbit appears at the case of $p / q=50 / 379 \approx 1 / 7.580$ [Fig. 7(e)], as shown in Fig. 4, the lower subcluster in Figs. 7(c)-7(e) seems to be related to the MB3 orbit.

Between Figs. 7(a) and 7(e), the total number of magnetic energy bands around $-7 \mathrm{eV}$ decreases with $B$ gradually. Further increase of $B$ leads to a further decrease of the number [Figs. 7(f) and $7(\mathrm{~g})$ ]. This would be related to the fact that energy states originating from the MB1 orbit disappear when their energy levels go up. If energy states originating from MB1 disappear, then the hybridized energy states related to the MB1 orbit also disappear.

While magnetic energy bands around $-7 \mathrm{eV}$ are disappearing, the cluster of magnetic energy bands appears around $-12 \mathrm{eV}$, as shown in Figs. 7(a)-7(h). In this lower cluster, one can find magnetic energy bands that go up with increasing $B$. They are expected to originate from the MB1 orbit. At the magnetic field of $p / q=48 / 397(\approx 1 / 8.271)$ [Fig. 7(h)], the Fermi energy goes down from the second lowest cluster to the lowest cluster, so that a large oscillation peak related to the MB1 orbit appears, as shown in Fig. 4. Thus, MB oscillations of MB1 are generated by the appearance and disappearance of magnetic energy bands that go up with increasing $B$. This is another piece of evidence for identifying these magnetic energy bands as energy states originating from the MB1 orbit.

Next, we shall comment on the MB oscillation related to the MB2 orbit. Figure 6(a) corresponds to magnetic energy bands in the case of $p / q \approx 1 / 7.983$ at which the MB oscillation related to the MB2 orbit occurs weakly, as shown in Fig. 4. It is difficult from Fig. 6(a) to identify magnetic energy bands originating from the MB2 orbit clearly. This is because the $\mathrm{MB}$ oscillation related to the MB2 orbit is weak [33] for the present system, as shown in Fig. 2. However, we can say that some magnetic energy bands cause the MB oscillation related to the MB2 orbit in a similar way to other MB oscillations because the Fermi energy exhibits a spikelike change at the magnetic field, as shown in Fig. 4. In addition, as shown in Fig. 6(a), the energy width of the cluster becomes maximum at the magnetic field. Therefore, we can also say that when the energy width of the cluster becomes maximum, the weak MB oscillation related to MB2 appears. These two facts would be key points to reveal the mechanism of the weak MB oscillation related to the $\mathrm{MB} 2$ orbit.

At the end of this section, we give a brief comment on a more quantitative description of the subcluster. As mentioned in Refs. [18,29-31], the magnetic energy bands depend strongly on the value of the rational number $p / q$. In the case of $B \propto p / q, p$ magnetic energy bands lie within a small energy range in the magnetic Brillouin zone [29]. In addition, as is discussed in Ref. [31], the set of $p$ magnetic energy bands corresponds to a cluster in many cases. To give a more quantitative description of the subclusters, we need to know 


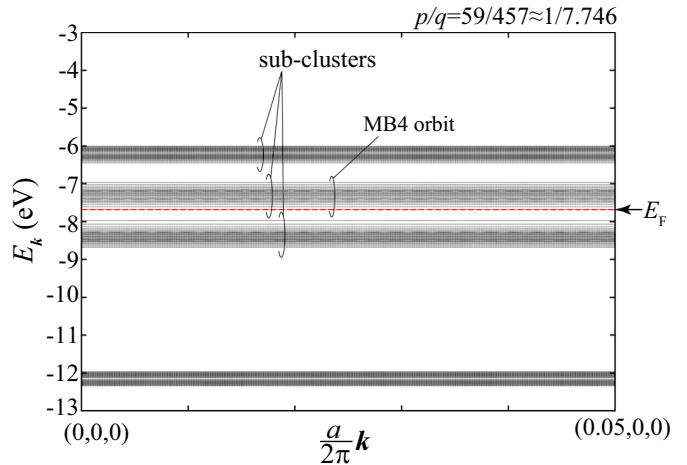

(a)

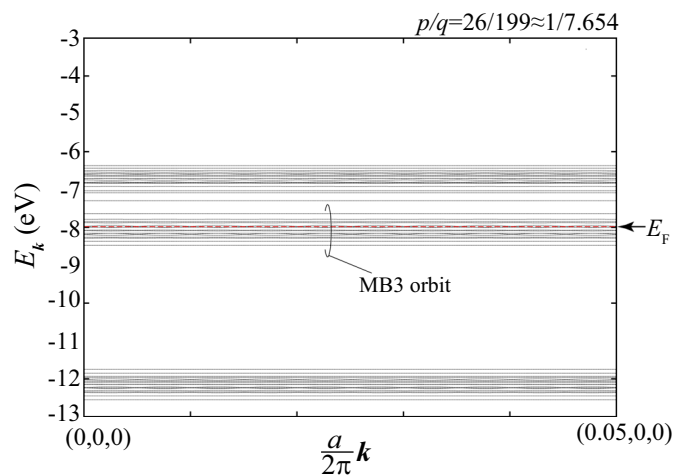

(c)

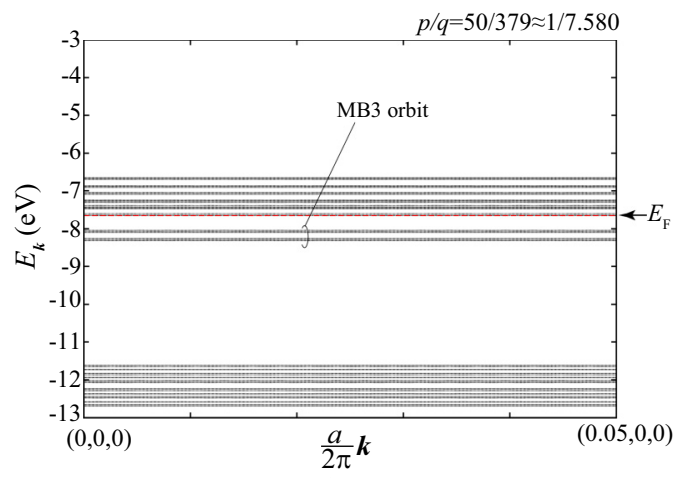

(e)

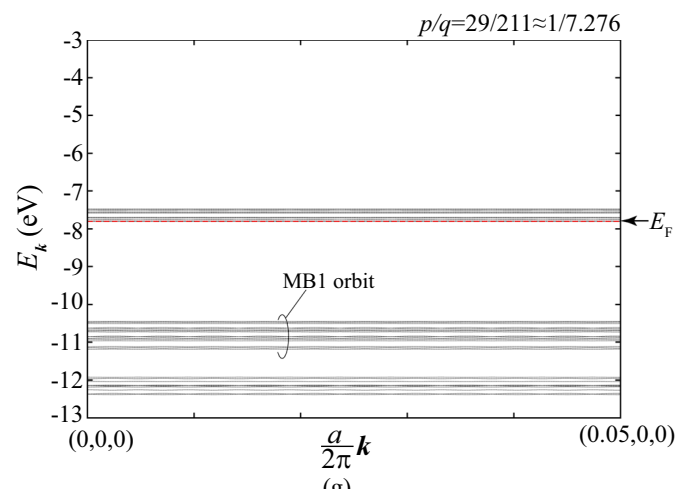

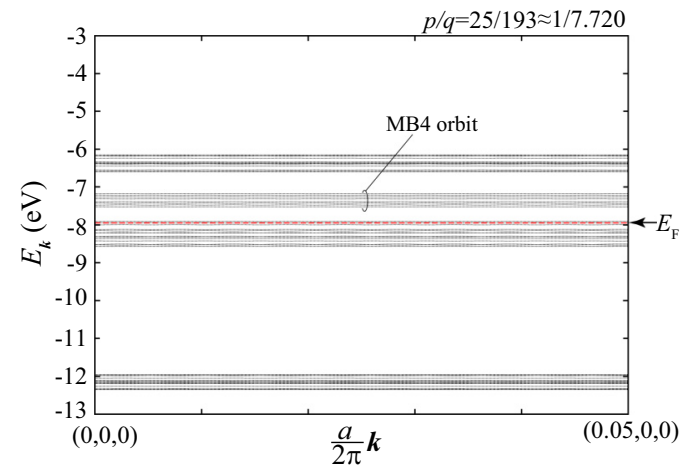

(b)

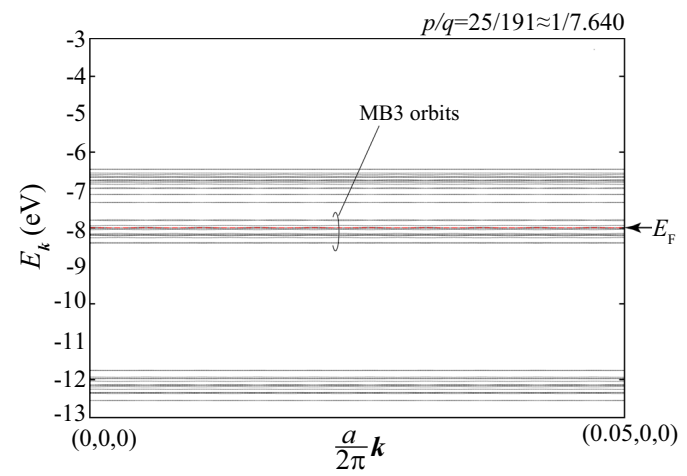

(d)

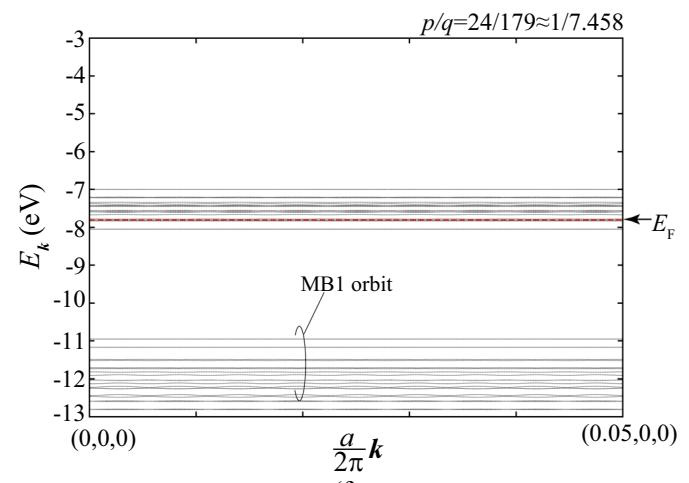

(f)

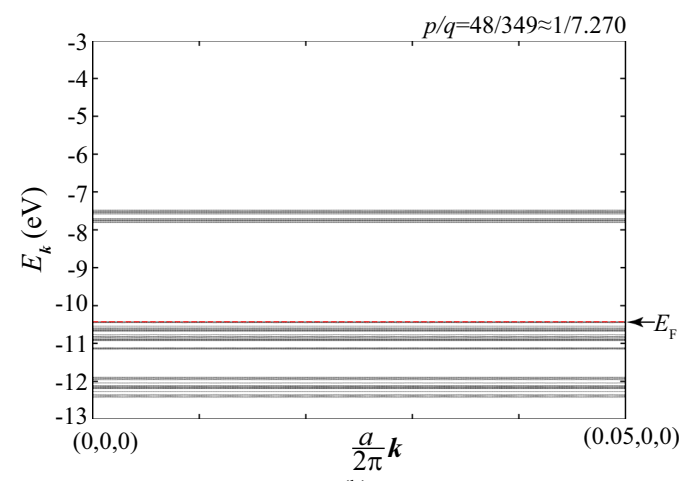

(h)

FIG. 7. Magnetic-field dependence of magnetic energy bands for the higher magnetic-field case, the $p / q$ values of which are (a) $59 / 457(\approx 1 / 7.746)$, (b) $25 / 193(\approx 1 / 7.720)$, (c) $26 / 199(\approx 1 / 7.654)$, (d) $25 / 191(\approx 1 / 7.640),($ e) $50 / 379(\approx 1 / 7.580),($ f) $24 / 179(\approx 1 / 7.458)$, (g) $29 / 211(\approx 1 / 7.276)$, and (h) $48 / 349(\approx 1 / 7.270)$, respectively. The position of the Fermi energy $\left(E_{F}\right)$ for the case of $\eta=0.52$ is indicated by the red line and arrow. 
what determines the largeness of the energy gaps between $p$ magnetic energy bands; this could be explored in future work.

\section{CONCLUDING REMARKS}

We investigate $\mathrm{MB}$ oscillations on the basis of magnetic energy bands calculated by the MFRTB method. It is found that subclusters of magnetic energy bands are generated by the $\mathrm{MB}$ in the cluster that corresponds to the energy level of the semiclassical orbit lying on the constant-energy surface. The formation of the subcluster is similar to the fine energy-band structures that appear in the simple-cubic system, where MB occurs infrequently [31]. While the fine energy-band structures for the case of the simple-cubic system are caused by hybridization between semiclassical orbits lying on the constant energy surface [31], the subclusters are caused by hybridization between semiclassical orbits containing MB points, or between semiclassical orbits lying on the constant energy surface and semiclassical orbits containing MB points. It is found that whenever the subcluster becomes unoccupied or occupied with increasing magnetic field, the Fermi energy jumps up or down, which results in MB oscillations.

Finally, we mention the similarity between the formation of magnetic energy bands and that of energy bands of solids. It is well known that energy bands in solids are formed via electron hopping between atomic orbitals as atoms approach each other. On the other hand, in the formation of magnetic energy bands, the semiclassical orbit lying on the constant energy surface and semiclassical orbits containing MB points serve as the atomic orbital. That is, a semiclassical energy level that corresponds to a semiclassical orbit has an energy width that increases gradually with an increasing magnetic field due to electron hopping between semiclassical orbits. Since electron hopping between semiclassical orbits is enlarged with increasing magnetic field, the inverse of the magnetic field serves as the distance between atoms in the formation of the magnetic energy bands. As the energy-band structure depends on the material, the magnetic energy-band structure that contains the fine energy level structure, the cluster structure, and the subcluster structures depends on both the material and the magnetic field.

\section{ACKNOWLEDGMENT}

This work was partially supported by JSPS KAKENHI Grants No. JP26400354, No. JP26400397, and No. JP16H00916.

\section{APPENDIX: MFRTB METHOD AND ITS APPLICATION TO A TWO-DIMENSIONAL SQUARE LATTICE IMMERSED IN A MAGNETIC FIELD}

In this Appendix, for convenience, we briefly explain the MFRTB method [29-31] and its application to a twodimensional square lattice immersed in a magnetic field. In addition, we mention the calculation method of the total energy [30,31].

The Dirac equation for an electron that moves in both a uniform magnetic field and the periodic potential of the crystal is given by

$$
\begin{aligned}
& {\left[c \boldsymbol{\alpha} \cdot\{\boldsymbol{p}+e \boldsymbol{A}(\boldsymbol{r})\}+\beta m c^{2}+\sum_{n} \sum_{i} v_{a_{i}}\left(\boldsymbol{r}-\boldsymbol{R}_{n}-\boldsymbol{d}_{i}\right)\right]} \\
& \times \Phi_{\boldsymbol{k}}(\boldsymbol{r})=E(\boldsymbol{k}) \Phi_{\boldsymbol{k}}(\boldsymbol{r}),
\end{aligned}
$$

where $\boldsymbol{A}(\boldsymbol{r})$ and $v_{a_{i}}\left(\boldsymbol{r}-\boldsymbol{R}_{n}-\boldsymbol{d}_{i}\right)$ are the external vector potential of the uniform magnetic field $\boldsymbol{B}$ and the scalar potential caused by the nucleus of atom $a_{i}$ that is located at $\boldsymbol{R}_{n}+\boldsymbol{d}_{i}$. Vectors $\boldsymbol{R}_{n}$ and $\boldsymbol{d}_{i}$ denote the translation vector of the lattice and the vector specifying the position of atom $a_{i}$, respectively. In Eq. (A1), $c, e$, and $m$ denote the velocity of light, the elementary charge, and the rest mass of electrons, respectively, and the matrices $\alpha=\left(\alpha_{x}, \alpha_{y}, \alpha_{z}\right)$ and $\beta$ stand for the usual $4 \times 4$ matrices. The vector $\boldsymbol{k}$ is the wave vector that belongs to the magnetic first Brillouin zone [29-31]. In the MFRTB method, the wave function $\Phi_{k}(\boldsymbol{r})$ is expanded by means of relativistic atomic orbitals for atoms immersed in a uniform magnetic field:

$$
\Phi_{\boldsymbol{k}}(\boldsymbol{r})=\sum_{\xi} \sum_{n} \sum_{i} C_{\boldsymbol{k}}^{\xi}\left(\boldsymbol{R}_{n}+\boldsymbol{d}_{i}\right) \psi_{\xi}^{a_{i}, \boldsymbol{R}_{n}+\boldsymbol{d}_{i}}(\boldsymbol{r}),
$$

where $C_{\boldsymbol{k}}^{\xi}\left(\boldsymbol{R}_{n}+\boldsymbol{d}_{i}\right)$ is the expansion coefficient and $\psi_{\xi}^{a_{i}, \boldsymbol{R}_{n}+\boldsymbol{d}_{i}}(\boldsymbol{r})$ denotes the relativistic atomic orbital for the atom $a_{i}$ that is immersed in a uniform magnetic field. By neglecting both overlap integrals involving different centers and hopping integrals involving three different centers, matrix elements of the Hamiltonian are given by [29]

$$
\begin{aligned}
H_{\boldsymbol{R}_{m} j \eta, \boldsymbol{R}_{n} i \xi}= & \left(\varepsilon_{\xi}^{a_{i}, \mathbf{0}}+\Delta \varepsilon_{\xi}^{a_{i}, \boldsymbol{d}_{i}}\right) \delta_{\boldsymbol{R}_{m}, \boldsymbol{R}_{n}} \delta_{j, i} \delta_{\eta, \xi}+\left(1-\delta_{\boldsymbol{R}_{m}, \boldsymbol{R}_{n}} \delta_{j, i}\right) e^{-i \frac{e B}{\hbar}\left(R_{n x}+d_{i x}-R_{m x}-d_{j x}\right)\left(R_{m y}+d_{j y}\right)} \\
& \times T_{\eta \xi}^{a_{j} a_{i}}\left(\boldsymbol{R}_{n}-\boldsymbol{R}_{m}+\boldsymbol{d}_{i}-\boldsymbol{d}_{j}\right)
\end{aligned}
$$

with

$$
\begin{gathered}
T_{\eta \xi}^{a_{j} a_{i}}\left(\boldsymbol{R}_{l}+\boldsymbol{d}_{i}-\boldsymbol{d}_{j}\right)=\int \psi_{\eta}^{a_{j}, \mathbf{0}}(\boldsymbol{r})^{\dagger} \frac{v_{a_{j}}(\boldsymbol{r})+v_{a_{i}}\left(\boldsymbol{r}-\boldsymbol{R}_{l}-\boldsymbol{d}_{i}+\boldsymbol{d}_{j}\right)}{2} \psi_{\xi}^{a_{i}, \boldsymbol{R}_{l}+\boldsymbol{d}_{i}-\boldsymbol{d}_{j}}(\boldsymbol{r}) d^{3} r, \\
\Delta \varepsilon_{\xi}^{a_{i}, \boldsymbol{d}_{i}}=\int \psi_{\xi}^{a_{i}, \boldsymbol{d}_{i}}(\boldsymbol{r})^{\dagger}\left\{\begin{array}{l}
\left\{\sum_{\boldsymbol{R}_{m}} \sum_{k} v_{a_{k}}\left(\boldsymbol{r}-\boldsymbol{R}_{m}-\boldsymbol{d}_{k}\right)\right\} \psi_{\xi}^{a_{i}, \boldsymbol{d}_{i}}(\boldsymbol{r}) d^{3} r, \\
\left(\boldsymbol{R}_{m}+\boldsymbol{d}_{k} \neq \boldsymbol{d}_{i}\right)
\end{array}\right.
\end{gathered}
$$


where $T_{\eta \xi}^{a_{j} a_{i}}\left(\boldsymbol{R}_{l}+\boldsymbol{d}_{i}-\boldsymbol{d}_{j}\right), \varepsilon_{\xi}^{a_{i}, \mathbf{0}}$, and $\Delta \varepsilon_{\xi}^{a_{i}, \boldsymbol{d}_{i}}$ denote the magnetic hopping integral, the atomic spectrum, and the energy of the crystal field for the nonzero magnetic-field case, respectively. To calculate $T_{\eta \xi}^{a_{j} a_{i}}\left(\boldsymbol{R}_{l}+\boldsymbol{d}_{i}-\boldsymbol{d}_{j}\right), \varepsilon_{\xi}^{a_{i}, \boldsymbol{0}}$, and $\Delta \varepsilon_{\xi}^{a_{i}, \boldsymbol{d}_{i}}$, the perturbation theory is employed in the MFRTB method [29]. This enables us to approximately express $T_{\eta \xi}^{a_{j} a_{i}}\left(\boldsymbol{R}_{l}+\boldsymbol{d}_{i}-\boldsymbol{d}_{j}\right)$, $\varepsilon_{\xi}^{a_{i}, \boldsymbol{0}}$, and $\Delta \varepsilon_{\xi}^{a_{i}, \boldsymbol{d}_{i}}$ by using the hopping integral, the atomic spectrum, and the energy of the crystal field for the zero-magnetic-field case [29]. The resultant matrix elements in the MFRTB method are given by

$$
\begin{aligned}
H_{\boldsymbol{R}_{m} j\left(n^{\prime} l^{\prime} J^{\prime} M^{\prime}\right), \boldsymbol{R}_{n} i(n l J M)}= & \left(\bar{\varepsilon}_{n l J}^{a_{i}}(\boldsymbol{B}=0)+\Delta \bar{\varepsilon}_{n l J M}^{a_{i}, \boldsymbol{d}_{i}}(\boldsymbol{B}=0)+\frac{e B}{2 m} \frac{2 J+1}{2 l+1} \hbar M\right) \delta_{\boldsymbol{R}_{m}, \boldsymbol{R}_{n}} \delta_{j, i} \delta_{n^{\prime} l^{\prime} J^{\prime} M^{\prime}, n l J M} \\
& +\left(1-\delta_{\boldsymbol{R}_{m}, \boldsymbol{R}_{n}} \delta_{j, i}\right) e^{-i \frac{e B}{2 \hbar}\left(R_{n x}+d_{i x}-R_{m x}-d_{j x}\right)\left(R_{n y}+d_{i y}+R_{m y}+d_{j y}\right)} t_{n^{\prime} l^{\prime} J^{\prime} M^{\prime}, n l J M}^{a_{j} a_{i}}\left(\boldsymbol{R}_{n}-\boldsymbol{R}_{m}+\boldsymbol{d}_{i}-\boldsymbol{d}_{j}\right)
\end{aligned}
$$

where $t_{n^{\prime} l^{\prime} J^{\prime} M^{\prime}, n l J M}^{a_{j} a_{i}}\left(\boldsymbol{R}_{n}-\boldsymbol{R}_{m}+\boldsymbol{d}_{i}-\boldsymbol{d}_{j}\right)$ denotes the relativistic hopping integral for the zero-magnetic-field case, and they are calculated by using the relativistic version of the Slater-Koster table [29]. In Eq. (A6), $\bar{\varepsilon}_{n \ell J}^{a_{i}}(\boldsymbol{B}=0)$ and $\Delta \bar{\varepsilon}_{n l J M}^{a_{i}, \boldsymbol{d}_{i}}(\boldsymbol{B}=0)$ represent the energy spectrum and the energy of the crystal field for the zero-magnetic-field case, respectively. The subscripts $n, \ell, J$, and $M$ are the principal, orbital, total angular momentum, and magnetic quantum numbers, respectively.

In this paper, we apply Eq. (A6) to a two-dimensional square lattice immersed in a uniform magnetic field, and we suppose that each lattice point has one atom with one $s$-electron, i.e., $(n, l, J, M)=(n, 0,1 / 2, \pm 1 / 2)$. Taking only the hopping integrals between nearest-neighbor atoms into consideration, and using Eq. (A6), we have the simultaneous equations for expansion coefficients as follows [29]:

$$
\begin{aligned}
& {\left[E_{0}+\frac{e B}{m} \hbar M+2 t \cos \left\{2 \pi\left(k_{x}+I \frac{p}{q}\right)\right\}\right] C_{k}^{n 0 \frac{1}{2} M}\left(\text { Ia e }_{y}\right)+t\left[C_{\boldsymbol{k}}^{n 0 \frac{1}{2} M}\left[(I+1) a \boldsymbol{e}_{y}\right]+C_{\boldsymbol{k}}^{n 0 \frac{1}{2} M}\left[(I-1) a \boldsymbol{e}_{y}\right]\right]} \\
& =E(\boldsymbol{k}) C_{\boldsymbol{k}}^{n 0 \frac{1}{2} M}\left({\text { I } \left.a \boldsymbol{e}_{y}\right),}\right.
\end{aligned}
$$

with

$$
\begin{gathered}
E_{0}=\bar{\varepsilon}_{n 0 \frac{1}{2}}^{a_{i}}(\boldsymbol{B}=0)+\Delta \bar{\varepsilon}_{n 0 \frac{1}{2} M}^{a_{i}, 0}(\boldsymbol{B}=0) \\
C_{\boldsymbol{k}}^{n^{\prime} 0 \frac{1}{2} M^{\prime}}\left[\left(I^{\prime}+1\right) a \boldsymbol{e}_{y}\right]=\left\{\begin{array}{c}
e^{-2 \pi k_{y}} C_{\boldsymbol{n}}^{n^{\prime} 0 \frac{1}{2} M^{\prime}}(0) \text { for } I^{\prime}=q-1, \\
C_{\boldsymbol{k}}^{n^{\prime} 0 \frac{1}{2} M^{\prime}}\left[\left(I^{\prime}+1\right) a \boldsymbol{e}_{y}\right] \text { for } I^{\prime} \neq q-1,
\end{array}\right. \\
C_{\boldsymbol{k}}^{n^{\prime} 0 \frac{1}{2} M^{\prime}}\left[\left(I^{\prime}-1\right) a \boldsymbol{e}_{y}\right]=\left\{\begin{array}{cc}
e^{2 \pi i k_{y}} C_{\boldsymbol{k}^{\prime} 0 \frac{1}{2} M^{\prime}}^{n^{\prime}}\left[(q-1) a \boldsymbol{e}_{y}\right] \text { for } I^{\prime}=0, \\
C_{\boldsymbol{k}}^{n^{\prime} M^{\prime}}\left[\left(I^{\prime}-1\right) a \boldsymbol{e}_{y}\right] & \text { for } I^{\prime} \neq 0,
\end{array}\right.
\end{gathered}
$$

where $t$ is the relativistic TB parameter, which is denoted by $K_{1}\left(n 0 \frac{1}{2}, n 0 \frac{1}{2}\right)_{\frac{1}{2}}$ in Ref. [29]. In the derivation of Eqs. (A7)-(A10), the magnitude of the magnetic field is assumed to be given by

$$
B=\frac{2 \pi \hbar}{e a^{2}} \frac{p}{q}
$$

where $p$ and $q$ are relatively prime integers, and $a$ denotes the lattice constant [18,29-31]. By solving the simultaneous equations, we have $2 q$ energy eigenvalues for each $\boldsymbol{k}$, and we obtain $E$ - $\boldsymbol{k}$ curves for the system immersed in a magnetic field. In the present calculations, we use the following values for the lattice constant and relativistic TB parameters:

$$
\begin{aligned}
a & =0.543 \mathrm{~nm}, \\
E_{0} & =-12.1538 \mathrm{eV}, \\
t & =-1.7391 \mathrm{eV} .
\end{aligned}
$$

These values are the same as those used in Refs. [29-31].

Next, we briefly explain the method to calculate the total energy [30,31]. According to the theorem mentioned in Ref. [25], the total number of $\boldsymbol{k}$-points in the magnetic Brillouin zone is equal to the total number of magnetic primitive unit cells contained in the system. We calculate $E(\boldsymbol{k})$ for all $\boldsymbol{k}$-points under the assumption that $\boldsymbol{k}$-points are uniformly distributed in the magnetic Brillouin zone [29]. Then, we obtain the total energy by summing values of $E(\boldsymbol{k})$ in order from the lowest energy state until the total number of states reaches the total number of electrons. In the present calculations, we take $8 q^{2} \times 10^{6}$ as the total number 
of $\boldsymbol{k}$-points [30,31]. This value is determined by requiring that the dependence of the total energy per unit volume on the volume of the system is negligibly small $[30,31]$.

[1] W. J. de Haas and P. M. van Alphen, Proc. Netherlands Roy. Acad. Sci. 33, 680 (1930).

[2] W. J. de Haas and P. M. van Alphen, Proc. Netherlands Roy. Acad. Sci. 33, 1106 (1930).

[3] W. J. de Haas and P. M. van Alphen, Proc. Netherlands Roy. Acad. Sci. 35, 454 (1932).

[4] D. Shoenberg, Magnetic Oscillation in Metals (Cambridge University Press, Cambridge, 1984).

[5] L. W. Shubnikov and W. J. de Haas, Proc. Netherlands Roy. Acad. Sci. 33, 130 (1930); 33, 163 (1930).

[6] L. Onsager, Philos. Mag. 43, 1006 (1952).

[7] I. M. Lifshitz and A. M. Kosevich, Sov. Phys. JETP 2, 636 (1956).

[8] N. W. Ashcroft and N. D. Mermin, Solid State Physics (Saunders, Philadelphia, 1976), Chap. 14.

[9] J. Kübler, Theory of Itinerant Electron Magnetism (Oxford University Press, Oxford, 2000), Chap. 1.

[10] M. H. Cohen and L. M. Falicov, Rhys. Rev. Lett. 7, 231 (1961).

[11] A. B. Pippard, Proc. R. Soc. London, Ser. A 270, 1 (1962); 287, 165 (1965).

[12] A. B. Pippard, Philos. Trans. R. Soc. London, Ser. A 256, 317 (1964).

[13] A. B. Pippard, in The Physics of Metals: 1, Electrons, edited J. M. Ziman (Cambridge University Press, Cambridge, 1969), p. 113.

[14] R. W. Stark and L. M. Falicov, Prog. Low Temp. Phys. 5, 235 (1967).

[15] J. Y. Fortin and T. Ziman, Phys. Rev. Lett. 80, 3117 (1998).

[16] M. Nakano, J. Phys. Soc. Jpn. 66, 19 (1997).

[17] A. S. Alexandrov and A. M. Bratkovsky, Phys. Lett. A 234, 53 (1997).

[18] D. R. Hofstadter, Phys. Rev. B 14, 2239 (1976).
[19] K. Machida, K. Kishigi, and Y. Hori, Phys. Rev. B 51, 8946 (1995).

[20] K. Kishigi, J. Phys. Soc. Jpn. 66, 910 (1997).

[21] S. Y. Han, J. S. Brooks, and J. H. Kim, Phys. Rev. Lett. 85, 1500 (2000).

[22] K. Kishigi and Y. Hasegawa, Phys. Rev. B 65, 205405 (2002).

[23] O. Gat and J. E. Avron, Phys. Rev. Lett. 91, 186801 (2003).

[24] O. Gat and J. E. Avron, New J. Phys. 5, 44 (2003).

[25] M. Taut, H. Eschrig, and M. Richter, Phys. Rev. B 72, 165304 (2005).

[26] V. M. Gvozdikov and M. Taut, Phys. Rev. B 75, 155436 (2007).

[27] W. H. Xu, L. P. Yang, M. P. Qin, and T. Xiang, Phys. Rev. B 78, 241102(R) (2008).

[28] K. Kishigi and Y. Hasegawa, Phys. Rev. B 90, 085427 (2014).

[29] K. Higuchi, D. B. Hamal, and M. Higuchi, Phys. Rev. B 91, 075122 (2015)

[30] D. B. Hamal, M. Higuchi, and K. Higuchi, Phys. Rev. B 91, 245101 (2015).

[31] M. Higuchi, D. B. Hamal, and K. Higuchi, Phys. Rev. B 95, 195153 (2017).

[32] P. G. Harper, Proc. Phys. Soc. London, Sect. A 68, 874 (1955).

[33] The peak originating from the MB2 orbit is weak, as shown in Figs. 2-4. This tendency is consistent with previous works $[25,26]$. According to the discussion mentioned in Sec. IV, the more occupied (unoccupied) states become unoccupied (occupied) states, the larger is the magnetization that would occur. In other words, the strength of the peak in Figs. 2-4 depends on the number of states going around the MB2 orbit. However, it will be left for future work to explore why the weak peak originates from the MB2 orbit, in addition to identifying the magnetic energy bands originating from the MB2 orbit. 